\title{
Comparison of the Pregnancy and Neonatal Outcomes of ICSI Performed With Frozen Versus Fresh TESA Sperm in Patients With Obstructive Azoospermia: a Retrospective Study
}

\section{Shenghao Wu}

The Second Affiliated Hospital and Yuying Children's Hospital of Wenzhou Medical University https://orcid.org/0000-0003-1380-7586

\section{Weiting Xia}

Wenzhou Medical University First Affiliated Hospital: The First Affiliated Hospital of Wenzhou Medical University

\section{Xin-Xin Xu}

Women's Hospital School of Medicine Zhejiang University

\section{Yanhong Wu}

The Second Affiliated Hospital and Yuying Children's Hospital of Wenzhou Medical University

\section{Yangyang Hu}

The Second Affiliated Hospital and Yuying Children's Hospital of Wenzhou Medical University

\section{Lizi Fang}

The Second Affiliated Hospital and Yuying Children's Hospital of Wenzhou Medical University Junzhao Zhao ( $\nabla$ z.joyce08@163.com )

The Second Affiliated Hospital and Yuying Children's Hospital of Wenzhou Medical University https://orcid.org/0000-0002-2385-205X

\section{Research}

Keywords: testicular sperm aspiration (TESA), obstructive azoospermia (OA), intracytoplasmic sperm injection (ICSI), pregnancy, newborn birth weight

Posted Date: August 23rd, 2021

DOl: https://doi.org/10.21203/rs.3.rs-824503/v1

License: (c) (1) This work is licensed under a Creative Commons Attribution 4.0 International License. Read Full License 


\section{Abstract}

\section{Background}

It is commonly believed that using frozen sperms after TESA was effective for OA patients. Nevertheless, scholars are worried about the prognosis of ICSI with frozen testicular sperm. In this study, we aim to compare the pregnancy and neonatal outcomes of ICSI using cryopreserved versus fresh spermatozoa collected by TESA.

\section{Methods}

A total of 317 cases of OA patients treated with ICSI in a university affiliated hospital from January 2016 to December 2020 were included in this retrospective study, and they were divided into two groups according to the sperm used for ICSI: Frozen sperm group $(\mathrm{N}=154)$ and Fresh sperm group $(\mathrm{N}=163)$. The outcomes were measured by the following indicators: Two pronucleus (2PN) fertilization rate, 2PN cleavage rate, high-quality blastocyst rate, average number of embryos transferred, implantation rate, clinical pregnancy rate, multiple pregnancy rate, miscarriage rate, preterm birth rate, live birth rate (LBR), sex ratio at birth (male) and average newborn birth weight.

\section{Results}

The present data showed no statistically significant difference in 2PN fertilization rates, 2PN cleavage rates, high-quality blastocyst rates and the average number of embryos transferred in the two groups. Similarly, no difference was found in implantation rate, clinical pregnancy rate, multiple pregnancy rate, miscarriage rate, premature delivery rate, LBR and sex ratio at birth $(P>0.05)$. The average newborn birth weight was similar in the two groups $(2932.61 \pm 728.40$ vs $3100.32 \pm 515.64)(P>0.05)$, but there was a higher incidence of Low-Birth-Weight newborn in the frozen group $(20.91 \%$ vs $8.49 \%)(P<0.05)$.

\section{Conclusions}

As for men with obstructive azoospermia, the use of frozen testicular sperm by TESA was efficient. There was a similar pregnancy outcome of ICSI using frozen or fresh spermatozoa collected by TESA. However, it may lead to higher incidence of newborns of low birth weight, which needs further research based on larger samples.

\section{Background}

Infertility has become a global medical and social problem. In recent years, the prevalence of infertility has continued to rise in both developed and developing countries. Statistics indicated that half of infertility cases are due to male infertility. Azoospermia has been identified in $5 \%$ of infertile men, which can be classified as obstructive azospermia (OA) or nonobstructive azoospermia (NOA) [1-2]. Obstructive azoospermia is a common disease in reproductive andrology and accounts for $40 \%$ of azoospermia, which results from obstruction of the excurrent ducts at any location between the rete testis 
and the ejaculatory ducts [3]. OA used to be a difficult problem in male infertility, and for these patients, sperm donation was the only option, which was despairing for the infertile families. Craft et al. [4] reported in 1997 that intracytoplasmic sperm injection(ICSI) using testis sperm by TESA for assisted reproduction is a feasible way to deal with obstructive azospermia. Since then, this method has been widely used, especially for patients with obstructive azoospermia, given its advantages of repeatability, low incidence of complications (bleeding or infections) and excellent success rate of sperm retrieval (96$100 \%$ [5]. However, due to the failure rate of IVF, these patients with OA often have to go through a second or third puncture. In order to minimize the damage caused by repeated puncture, many institutions have tried to freeze the sperm obtained by diagnostic testicular puncture. Is there any difference between frozen or fresh testicular sperm? It is still controversial about the pregnancy outcomes of ICSI using frozen or fresh testicular sperm. In this study, the use of frozen and fresh testicular sperm for ICSI were compared, in terms of pregnancy and birth outcomes in patients with OA.

\section{Methods}

\section{Study design and patient selection criteria}

The study was approved by the Independent Ethics Committee of The Second Affiliated Hospital and Yuying Children's Hospital of Wenzhou Medical University (nunber: 2021-K-74-02). A total of 317 cases of OA patients (female partner age $\leq 40$ years old) treated with ICSI in our center from January 2016 to December 2020 were included in this retrospective comparative study. Among them, men who agreed to freeze their sperms by diagnostic TESA for subsequent ART were included in the "Frozen sperm group" $(\mathrm{N}$ $=154$ ), and the other 163 cases which used fresh sperms (obtained by TESA on the day of oocyte retrival) were included as fresh sperm group. The two groups were matched in age and included all consecutive men who met the above criteria. None of the participants had abnormal results of chromosome analysis and sex hormone tests. The detailed flow chart is shown in Fig. 1.

\section{Treatment Regimen}

\section{Testicular sperm aspiration (TESA)}

The testicle was anesthetized locally using $2 \%$ lidocaine. A 23-gauge needle connected with a 5-ml syringe containing 5\% G-MOPS medium (Vitrolife, SanDiego, USA) was used to insert into the testis through the skin of the scrotum to obtain enough testicular tissue. Immediately after the collection, the testicular tissue had been placed in a dish filled with HEPES- buffered medium (G-MOPS) and sent to the laboratory for microscopy. The obtained testicular tissue was minced with a sterile empty needle, and the suspension was examined for sperm morphological quality and motility under microscope $(\times 400)$.

\section{Sperm freezing and thawing}

Sperm freezing medium (SAGE, CooperSurgical, USA) was added to the semen with the same volume after preheating it to room temperature. The solution was carefully mixed after each addition. Each $0.6 \sim$ 
$0.8 \mathrm{ml}$ of mixed semen was put into a $1.5 \mathrm{ml}$ cryotube, and later placed in a refrigerator at $4^{\circ} \mathrm{C}$ for 30 minutes. Each tube was carefully transferred into a aluminum bracket, which was placed in a freezing cylinder. The cylinder was positioned in a liquid nitrogen storage tank for long-term storage.

When performing ICSI,the cryotubes were removed from the liquid nitrogen tank and placed at room temperature for 15-30 minutes. The samples were later on transferred to centrifuge tubes and centrifuged to concentrate the sperm cells. Morphological quality and motility of sperms were evaluated, and sperms of good quality were chosen for ICSI.

The ICSI was performed by using frozen-thawed testicular sperm or fresh testicular sperm. Two pronuclear oosperm counts and the embryo development stage on day 3, day 5 and day 6 after ICSI were observed and recorded. After that, blastocysts of good quality were selected according to the Gardner blastocyst grading system for intrauterine embryo transfer, and serum $\beta$-HCG was checked after 14 days to determine the pregnancy status. Clinical pregnancy was defined as the presence of an intrauterine gestational sac with a yolk sac, a fetal pole, and fetal heart pulsations at approximately 4 weeks after implantation. Live birth was referred to the birth of a live infant at $\geq 24$ weeks' gestation. The gender and birth weight of the newborns were collected after birth. Infants below 2500 grams will be classified as Low-Birth-Weight, while infants above 4000 grams as macrosomia.

\section{Statistical analysis}

Data analysis was performed with SPSS version 20.0 (IBM Corporation), and Student's t test was used to compare continuous variables. Chi-square test was performed for categorical variables. A $P$-value of < 0.05 was considered statistically significant.

\section{Results}

A total of 317 patients were included in this study, and they were divided into two groups according to the sperm types used for ICSI. In the frozen sperm group, the sperm samples from 154 men were cryopreserved after TESA. The fresh sperm group used freshly aspirated sperm samples from 163 matched patients with azoospermia. The demographic, fertility-related, and ICSI characteristics of the two groups were similar (Table 1). There were no significant differences in terms of female's age, male's age, duration of infertility, the preoperative sex hormone test results, body mass index (BMI), rates of smoking and alcohol drinking between the two groups. 
Table 1

Baseline characteristics of the two groups

\begin{tabular}{|llll|}
\hline Characteristics & Frozen sperm( $\mathbf{n = 1 5 4 )}$ & Fresh sperm( $\mathbf{n = 1 6 3 )}$ & $P$-value \\
\hline Female age(years) & $29.67 \pm 4.80$ & $30.02 \pm 5.09$ & 0.523 \\
\hline Male age(years) & $32.39 \pm 5.23$ & $33.40 \pm 6.19$ & 0.117 \\
\hline Infertility(years) & $4.01 \pm 3.19$ & $4.03 \pm 3.29$ & 0.947 \\
\hline LH(IU/L) & $4.9 \pm 2.3$ & $5.0 \pm 2.3$ & 0.929 \\
\hline FSH(IU/L) & $8.8 \pm 4.7$ & $9.8 \pm 5.0$ & 0.070 \\
\hline Prolactin (ng/ml) & $9.0 \pm 4.3$ & $9.7 \pm 4.4$ & 0.162 \\
\hline Estradiol (pg/ml) & $27.3 \pm 8.1$ & $27.4 \pm 8.4$ & 0.912 \\
\hline Testosterone (ng/ml) & $5.2 \pm 1.8$ & $4.9 \pm 1.7$ & 0.139 \\
\hline BMI(kg/m $\left.{ }^{2}\right)$ & $21.8 \pm 1.9$ & $22.1 \pm 1.6$ & 0.193 \\
\hline Smoking rate(\%) & $50.65 \%(78 / 154)$ & $54.60 \%(89 / 163)$ & 0.496 \\
\hline Alcohol rate(\%) & $47.40 \%(73 / 154)$ & $49.08 \%(80 / 163)$ & 0.089 \\
\hline Note LH: Luteinizing hormone;FS: Follicle Stimulating hormone; BMl: Body Mass Index. & \\
\hline
\end{tabular}

The ICSI outcomes were similar, with no difference in 2PN fertilization rates, 2PN cleavage rates and highquality blastocyst rates $(P>0.05)$. The specific results are shown in Table 2.

Table 2

Comparison of the outcomes of ICSI between the two groups

\begin{tabular}{|llll|}
\hline Outcomes & Frozen sperm( $\mathrm{n}=154)$ & Fresh sperm( $\mathrm{n}=163)$ & $P$-value \\
\hline 2PN Fertilization rate(\%) & $81.21 \%(1297 / 1597)$ & $83.30 \%(1267 / 1521)$ & 0.128 \\
\hline 2PN Cleavage rate(\%) & $97.07 \%(1259 / 1297)$ & $96.92 \%(1228 / 1267)$ & 0.826 \\
\hline High-quality blastocyst rate(\%) & $44.62 \%(232 / 520)$ & $44.76 \%(235 / 525)$ & 0.962 \\
\hline Note $P$-value by ANOVA, Chi square test. Significance: $P<0.05$ & \\
\hline
\end{tabular}

For the pregnancy outcomes, the clinical pregnancy rate was $57.28 \%$ and the live birth rate was $44.17 \%$ in the frozen group, and they were $58.05 \%$ and $45.37 \%$ in the fresh group respectively, while the average number of embryos transferred was 1.84 and 1.79. There was no difference in the average number of embryos transferred, implantation rate, clinical pregnancy rate and live birth rate, and also the 
miscarriage rate, multiple pregnancy rate, preterm birth rate, and sex ratio at birth (male) were similar in the two groups (Table 3).

Table 3

Comparison of pregnancy outcomes between the two groups

\begin{tabular}{|c|c|c|c|c|}
\hline Outcomes & $\begin{array}{l}\text { Frozen sperm( } n= \\
154)\end{array}$ & $\begin{array}{l}\text { Fresh sperm }(n= \\
163)\end{array}$ & $\chi^{2}$ & $\begin{array}{l}P \\
\text { value }\end{array}$ \\
\hline $\begin{array}{l}\text { Average number of embryos } \\
\text { transferred }\end{array}$ & $1.84 \pm 0.93$ & $1.79 \pm 0.96$ & & 0.511 \\
\hline Implantation rate(\%) & $43.28 \%(145 / 335)$ & $42.39 \%(142 / 335)$ & 0.055 & 0.815 \\
\hline Clinical pregnancy rate(\%) & $57.28 \%(118 / 206)$ & $58.05 \%(119 / 205)$ & 0.025 & 0.875 \\
\hline Multiple pregnancy rate(\%) & $24.58 \%(29 / 118)$ & $19.33 \%(23 / 119)$ & 0.953 & 0.329 \\
\hline Miscarriage rate(\%) & $12.71 \%(15 / 118)$ & $12.61 \%(15 / 119)$ & 0.001 & 0.980 \\
\hline Preterm birth rate(\%) & $10.17 \%(12 / 118)$ & $5.88 \%(7 / 119)$ & 1.477 & 0.224 \\
\hline Live birth rate(\%) & $44.17 \%(91 / 206)$ & $45.37 \%(93 / 205)$ & 0.059 & 0.808 \\
\hline Sex ratio at birth(\%) & $96.43 \%(54 / 56)$ & $92.73 \%(51 / 55)$ & 0.021 & 0.886 \\
\hline Average newborn birth weight(g) & $2932.61 \pm 728.40$ & $3100.32 \pm 515.64$ & & 0.052 \\
\hline $\begin{array}{l}\text { Incidence of Low-Birth-Weight } \\
\text { newborns }(\%)\end{array}$ & $20.91 \%(23 / 110)$ & $8.49 \%(9 / 106)$ & 6.597 & 0.010 \\
\hline
\end{tabular}

By the end of follow-up date, there were eleven women still in pregnancy in both groups. 110 babies were born in the frozen sperm group, of which 54 were males and 56 were females, including 23 low birth weight newborns and 5 macrosomias. Correspondingly, a total of 106 babies were born in the fresh sperm group, including 51 males and 55 females, 9 low birth weight newborns, and 4 macrosomias. The average birth weight of newborns between the two groups was similar $(P>0.05)$, but we found that there was a higher incidence of low birth weight newborn in the frozen sperm group $(20.91 \%$ vs $8.49 \%)(P$ $<0.05$, Fig. 2, Table 3)

\section{Discussion}

Although a lot of efforts have been made for helping the azoospermia patients, there are still $20 \%-30 \%$ unable to obtain sperm suitable for injection. Therefore, repeated punctures were performed to increase the success rate of sperm acquisition in most centers. It was shown that repeated testicular or epididymal puncture has no remarkable effect on the outcomes of pregnancy [6]. However, multiple operations may cause damage to the blood supply of the testicular seminiferous tubules, produce hematoma formation 
in the testis, and bring about fibrosis of the testicular tissue and even testicular atrophy, which may lead to long-term complications such as male autoimmune response, osteoporosis, insulin resistance and depression [7]. In addition, some azoospermia patients may lose the opportunity to be a biological father in the event of failure of TESA on the day of oocyte collection, and thus have to choose insemination with donor spermatozoa or oocyte cryopreservation, if they did not undergo diagnostic TESA and sperm freezing in advance. Therefore, cryopreservation of testicular sperm is important for fertility preservation in azoospermia patients, the full and effective use of sperm is key to the treatment of obstructive azoospermia. It is commonly believed that freezing sperms after TESA was a safe, economical, and effective way for OA patients [8], and at the same time, the problem of damage mentioned above will be solved. Nevertheless, some scholars were still worried about the pregnancy outcomes of ICSI with frozen testicular sperm and recommended the use of fresh oocytes with fresh testicular sperm as the first-choice treatment [9-10]. Hauser et al.[10] proposed that the use of fresh testicular sperm results in a higher fertilization rate and clinical pregnancy rate than frozen testicular sperm. Therefore, the author insisted that fresh testicular sperm should be considered first for ICSI in patients with virtual azoospermia or cryptozoospermia because of their superior fertility. In 2015, a retrospective study including 110 cycles of TESE-ICSI were conducted by Park et al [11], who confirmed that statistically significant differences were observed in the pregnancy and implantation rates between fresh and frozen testicular spermatozoa group, in spite of similar laboratory outcomes, clinical pregnancy and delivery.

But analogous with the results of our study, most studies have suggested a similar results in terms of fertilization rate, cleavage rate and clinical pregnancy rate in ICSI with frozen testicular sperm [12-14]. OU L et al. [12] found that frozen testicular sperm or fresh testicular sperm extracted from patients with OA have the same $2 \mathrm{PN}$ fertilization and pregnancy potential $(74.41 \%$ vs $76.43 \%$ and $46.81 \%$ vs $53.39 \%$, respectively). Another retrospective study in 2018 suggested that freezing low-count sperm collected by TESA with a cryoprotectant was an efficient method. The clinical pregnancy rates of ICSI with fresh testicular sperm and frozen testicular sperm were $61.7 \%$ and $55.1 \%$, although the pregnancy rate in the fresh sperm group was higher, the difference was not significant. It shows that the two types of sperm are equally reliable for ICSI [15] .

In our study, in addition to focusing on the results of laboratory outcomes, clinical pregnancy and delivery, we further analysed the gender and birth weight of the newborns. There was a resemblance result of sex ratio and average newborn birth weight in the two groups, but in spite of this, the incidence of low-weight newborns significantly increased when frozen testicular sperm was used for ICSI (20.91\% vs $8.49 \%)$. The average birth weight of low-birth-weight newborns in the frozen and fresh sperm group was $1947 \mathrm{~g}$ and $2106 \mathrm{~g}$ respectively. Among them, 17 in the frozen sperm group were twin newborns, and 8 in the other group. To be more specific, in the frozen sperm group, two of the newborns were of very low birth weight (VLBW), and two infants have extremely low birth weight (ELBW)(Table 4). From what has been discussed above, we may reasonably arrive at the conclusion that the use of frozen sperm for ICSI may increase the incidence of low birth weight infants. LBW is one of the leading causes of neonatal and infant morbidity and mortality[16], which is also associated with long-term consequences including cerebral palsy, sensory deficits, cardiovascular and respiratory illnesses. One possible explanation is that 
resuscitation of frozen sperm has a negative effect on total count and motile count, and poor quality of semen may be related with low birth weight in the offspring [17]. This may also be due to the frozen damage of the sperm during the process of cooling, thawing and cryoprotectant exposure, which might result in changes in the function and structure of sperm [18], bringing about a higher rate of deformed sperm. Higher sperm deformity rate may be contributed to more chances of abnormal expression of imprinting genes, which will cause inefficient placental nutrient supply, resulting in reduction in neonatal birth weight [19]. The above situation is more prominent in multiple pregnancies. LBW is a known complication of multiple gestation [20]. A recently published research revealed that mothers with multiple pregnancies were at a significantly increased risk of delivering babies with LBW, both in the case of preterm and term birth [21]. In our study, the rate of multiple pregnancies in the frozen sperm group was $5 \%$ higher than the fresh group. Although there was no significant differences, this may be one of the reasons for the increased low birth weight rate in the freezing group. 
Table 4

Detailed Characteristics of Low-Birth-Weight newborns

\begin{tabular}{|c|c|c|c|c|c|c|}
\hline Case & sperm & $\begin{array}{l}\text { Female } \\
\text { age(years) }\end{array}$ & $\begin{array}{l}\text { Male } \\
\text { age(years) }\end{array}$ & Single/twins & $\begin{array}{l}\text { Birth } \\
\text { weight(g) }\end{array}$ & $\begin{array}{l}\text { Gestational } \\
\text { age(weeks) }\end{array}$ \\
\hline 1 & frozen & 34 & 34 & single & 1150 & 31 \\
\hline 2 & frozen & 31 & 31 & twins & 2400 & 37 \\
\hline 3 & frozen & 27 & 27 & single & 2200 & 37 \\
\hline 4 & frozen & 32 & 39 & twins & 2350 & 37 \\
\hline 5 & frozen & 32 & 36 & single & 2300 & 37 \\
\hline 6 & frozen & 40 & 44 & single & 1850 & 32 \\
\hline 7 & frozen & 27 & 42 & twins & 2400 & 36 \\
\hline 8 & frozen & 28 & 31 & twins & 2340 & 36 \\
\hline 9 & frozen & 28 & 31 & twins & 2440 & 36 \\
\hline 10 & frozen & 32 & 36 & twins & 2300 & 37 \\
\hline 11 & frozen & 31 & 36 & twins & 2200 & 39 \\
\hline 12 & frozen & 24 & 32 & single & 2400 & 37 \\
\hline 13 & frozen & 31 & 34 & twins & 1800 & 32 \\
\hline 14 & frozen & 31 & 34 & twins & 1600 & 32 \\
\hline 15 & frozen & 26 & 25 & twins & 1700 & 30 \\
\hline 16 & frozen & 26 & 25 & twins & 1400 & 30 \\
\hline 17 & frozen & 30 & 34 & twins & 700 & 34 \\
\hline 18 & frozen & 30 & 34 & twins & 700 & 34 \\
\hline 19 & frozen & 26 & 28 & twins & 2350 & 37 \\
\hline 20 & frozen & 37 & 42 & single & 2300 & 36 \\
\hline 21 & frozen & 31 & 33 & twins & 2490 & 38 \\
\hline 22 & frozen & 24 & 27 & twins & 1600 & 31 \\
\hline 23 & frozen & 24 & 27 & twins & 1800 & 31 \\
\hline 24 & fresh & 25 & 32 & twins & 1750 & 33 \\
\hline 25 & fresh & 30 & 33 & twins & 2100 & 36 \\
\hline
\end{tabular}




\begin{tabular}{|lllllll|}
\hline Case & sperm & $\begin{array}{l}\text { Female } \\
\text { age(years) }\end{array}$ & $\begin{array}{l}\text { Male } \\
\text { age(years) }\end{array}$ & Single/twins & $\begin{array}{l}\text { Birth } \\
\text { weight(g) }\end{array}$ & $\begin{array}{l}\text { Gestational } \\
\text { age(weeks) }\end{array}$ \\
\hline 26 & fresh & 30 & 33 & twins & 2250 & 36 \\
\hline 27 & fresh & 24 & 26 & twins & 2250 & 38 \\
\hline 28 & fresh & 24 & 26 & twins & 2100 & 38 \\
\hline 29 & fresh & 34 & 35 & twins & 1850 & 36 \\
\hline 30 & fresh & 29 & 33 & twins & 2450 & 37 \\
\hline 31 & fresh & 29 & 33 & twins & 2100 & 37 \\
\hline 32 & fresh & 31 & 34 & single & 2100 & 33 \\
\hline $\begin{array}{l}\text { Note There were 23 cases of Low-Birth-Weight newborns in the frozen sperm group (1-23), and 9 } \\
\text { cases in the fresh sperm group (24-32). }\end{array}$ \\
\hline
\end{tabular}

The risk of deformity is another problem for newborns after using testicular sperm. There were no cases of fetal or neonatal malformations found in this study. In a population-based cohort study, in which all Danish children were delivered after testicular sperm aspiration (TESA) and fresh embryo transfer in Denmark from 1995 to 2009, the outcome of newborns, including congenital abnormalities after ICSI treatment using epididymal or testicular sperm, has no difference with that after ICSI/IVF treatment using ejaculate sperm or natural conception [22]. A 10-year study in China focusing on the neonatal outcome of children born after ICSI with epididymal or testicular sperm clearly showed that freezing testicular sperm has no effect on the rate of neonatal deformity [23].

\section{Conclusion}

As for men with OA, the use of frozen testicular sperm by TESA was an efficient treatment. Testicular sperm cryopreservation is the best option available to preserve fertility in patients with azoospermia, which can avoid cancellation of in vitro fertilization cycles due to the failure of TESA on the day of oocyte collection. There was a similar pregnancy outcome of intracytoplasmic sperm injection (ICSI) using frozen or fresh spermatozoa collected by TESA. However it may lead to higher incidence of newborns of low birth weight when using frozen testicular sperm, which needs further research and discussion based on larger samples.

\section{Abbreviations}

ICSI: Intracytoplasmic sperm injection

IVF: In vitro fertilization

TESA: Testicular sperm aspiration 
OA: Obstructive azoospermia

NOA: Nonobstructive azoospermia

2PN: Two pronucleus

LBR: Live birth rate

HCG: Human chorionic gonadotropin

LH: Luteinizing hormone

FSH: Follicle Stimulating hormone

BMl: Body mass index

LBW: Low birth weight

VLBW: Very low birth weight

ELBW: Extremely low birth weight

\section{Declarations}

\section{Ethics approval and consent to participate}

The study was approved by the Independent Ethics Committee of The Second Affiliated Hospital and Yuying Children's Hospital of Wenzhou Medical University (nunber: 2021-K-74-02). All authors have given their consent for participation and publication of the study.All patients have signed the appropriate forms, for their inclusion in the study.

\section{Consent for publication}

All authors consent to publication of this manuscript.

\section{Availability of data and material}

The data can be shared upon request from the editorial team of the journal.

\section{Competing interests}

The authors report no conflict of interests.

\section{Funding}

This study is funded by Medicine Grant from Wenzhou Bureau of Science and Technology, Grant/Award Number: Y20180018. 


\section{Authors' contributions}

Shenghao Wu and Junzhao Zhao are responsible for the study conception and design. All authors contributed to patients' selection, data collection and statistical analysis. The first draft of the manuscript was written by Shenghao Wu and improved by all authors. All authors read and approved the final manuscript.

\section{Acknowledgements}

Not applicable.

\section{References}

1. Shuai HL, Ye Q, Huang YH, Xie BG. Comparison of conventional in vitro fertilisation and intracytoplasmic sperm injection outcomes in patients with moderate oligoasthenozoospermia. Andrologia. 2015;47(5):499-504.

2. Gudeman SR, Townsend B, Fischer K, Walters RC, Crain D. Etiology of azoospermia in a military population. The Journal of urology. 2015;193(4):1318-21.

3. Blok JM, van Roekel C, Oude Ophuis RJA, Lock T. Open epididymal spermatozoa aspiration for obstructive azoospermia. Andrologia. 2019;51(4):e13218.

4. Gorgy A, Podsiadly BT, Bates S, Craft IL. Testicular sperm aspiration (TESA): the appropriate technique. Human reproduction (Oxford, England). 1998;13(4):1111-3.

5. Cito G, Coccia ME, Sessa F, Cocci A, Verrienti P, Picone R, et al. Testicular Fine-Needle Aspiration for Sperm Retrieval in Azoospermia: A Small Step toward the Technical Standardization. World J Mens Health. 2019;37(1):55-67.

6. Talas $\mathrm{H}$, Yaman O, Aydos K. Outcome of repeated micro-surgical testicular sperm extraction in patients with non-obstructive azoospermia. Asian journal of andrology. 2007;9(5):668-73.

7. Donoso P, Tournaye H, Devroey P. Which is the best sperm retrieval technique for non-obstructive azoospermia? A systematic review. Human reproduction update. 2007;13(6):539-49.

8. Roque $M$, Valle M, Marques F, Sampaio M, Geber S. Intracytoplasmic sperm injection outcomes with cryopreserved testicular sperm aspiration samples. Andrologia. 2016;48(3):252-6.

9. Okuyama N, Obata R, Oka N, Nakamura Y, Hattori H, Nakajo Y, et al. Long-term clinical outcomes of testicular sperm extraction and intracytoplasmic sperm injection for infertile men. Reprod Med Biol. 2018;17(1):82-8.

10. Hauser R, Bibi G, Yogev L, Carmon A, Azem F, Botchan A, et al. Virtual azoospermia and cryptozoospermia--fresh/frozen testicular or ejaculate sperm for better IVF outcome? Journal of andrology. 2011;32(5):484-90.

11. Park YS, Lee SH, Lim CK, Cho JW, Yang KM, Seo JT. Effect of testicular spermatozoa on embryo quality and pregnancy in patients with non-obstructive azoospermia. Systems biology in 
reproductive medicine. 2015;61(5):300-6.

12. Ou L, Guo YH, Sun YP, Su YC. Outcomes of ICSI with microamount frozen-thawed sperm obtained by PESA or TESA in the treatment of azoospermia]. Zhonghua nan ke xue $=$ National journal of andrology. 2010;16(4):328-32.

13. Javed A, Ramaiah MK, Talkad MS. ICSI using fresh and frozen PESA-TESA spermatozoa to examine assisted reproductive outcome retrospectively. Obstetrics \& gynecology science. 2019;62(6):429-37.

14. Karacan M, Alwaeely F, Erkan S, Çebi Z, Berberoğlugil M, Batukan M, et al. Outcome of intracytoplasmic sperm injection cycles with fresh testicular spermatozoa obtained on the day of or the day before oocyte collection and with cryopreserved testicular sperm in patients with azoospermia. Fertility and sterility. 2013;100(4):975-80.

15. Yu G, Liu Y, Zhang H, Wu K. Application of testicular spermatozoa cryopreservation in assisted reproduction. International journal of gynaecology and obstetrics: the official organ of the International Federation of Gynaecology and Obstetrics. 2018;142(3):354-8.

16. Silveira MF, Victora CG, Horta BL, da Silva BGC, Matijasevich A, Barros FC, et al. Low birthweight and preterm birth: trends and inequalities in four population-based birth cohorts in Pelotas, Brazil, 19822015. Int J Epidemiol. 2019;48(Suppl 1):i46-i53.

17. Anderson RE, Hanson HA, Thai D, Zhang C, Presson AP, Aston KI, et al. Do paternal semen parameters influence the birth weight or BMI of the offspring? A study from the Utah Population Database. J Assist Reprod Genet. 2018;35(5):793-9.

18. Gómez-Torres MJ, Medrano L, Romero A, Fernández-Colom PJ, Aizpurúa J. Effectiveness of human spermatozoa biomarkers as indicators of structural damage during cryopreservation. Cryobiology. 2017;78:90-4.

19. Li B, Ma Y, Huang J, Xiao X, Li L, Liu C, et al. Probing the effect of human normal sperm morphology rate on cycle outcomes and assisted reproductive methods selection. PLoS One. 2014;9(11):e113392.

20. Keyhan S, Truong T, Li YJ, Jackson-Bey T, Eaton JL. Preterm Delivery and Low Birth Weight Among Neonates Conceived With Intracytoplasmic Sperm Injection Compared With Conventional In Vitro Fertilization. Obstet Gynecol. 2018;131(2):262-8.

21. Kasuga $Y$, Ikenoue $S$, Tamagawa M, Oishi M, Endo T, Sato $Y$, et al. What are the causes for low birthweight in Japan? A single hospital-based study. PLoS One. 2021;16(6):e0253719.

22. Fedder J, Loft A, Parner ET, Rasmussen S, Pinborg A. Neonatal outcome and congenital malformations in children born after ICSI with testicular or epididymal sperm: a controlled national cohort study. Human reproduction (Oxford, England). 2013;28(1):230-40.

23. Jin L, Li Z, Gu L, Huang B. Neonatal outcome of children born after ICSI with epididymal or testicular sperm: A 10-year study in China. Sci Rep. 2020;10(1):5145.

\section{Figures}




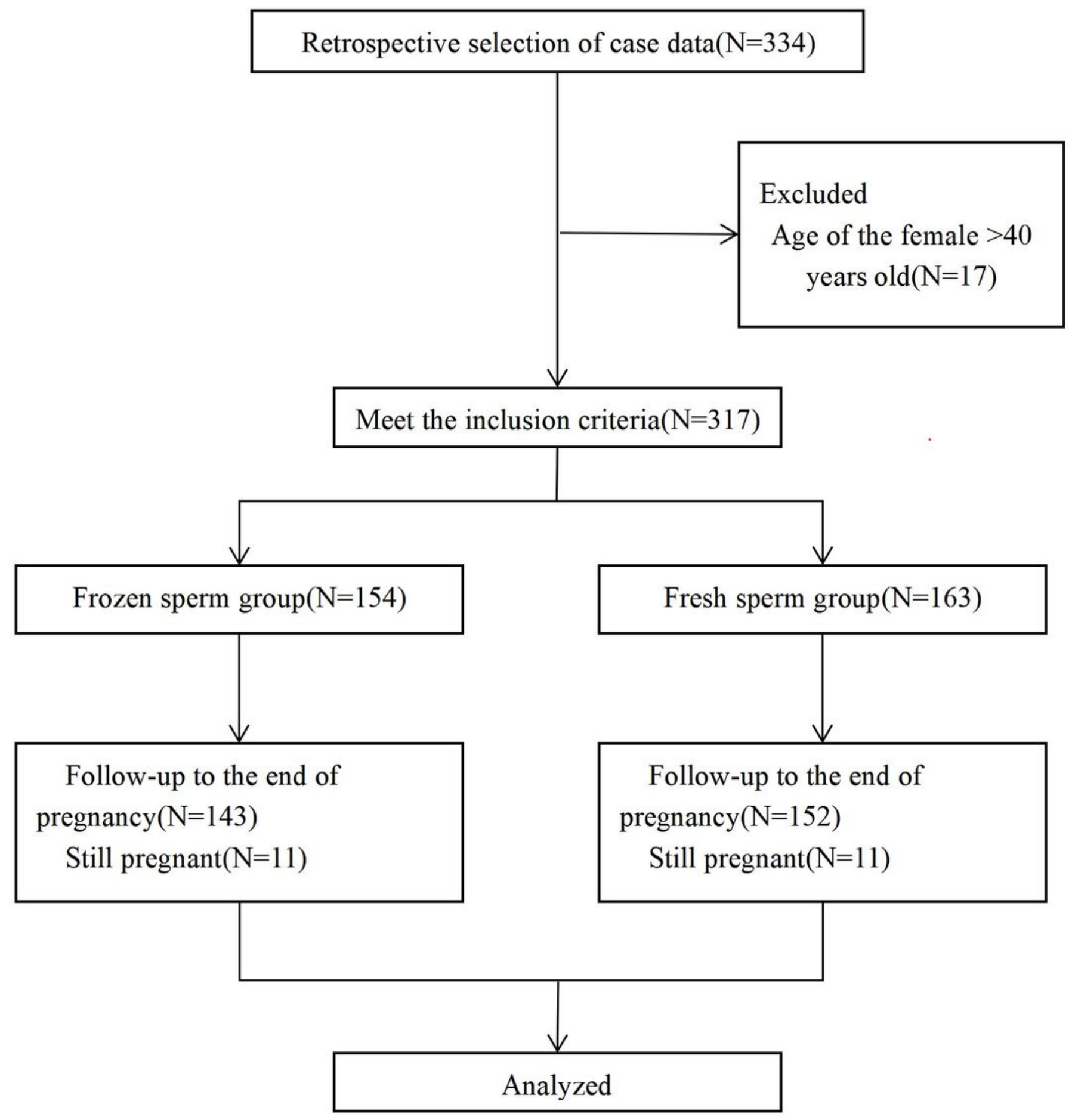

Figure 1

Flow chart showing the design, inclusion and exclusion criteria of patients in the study. 


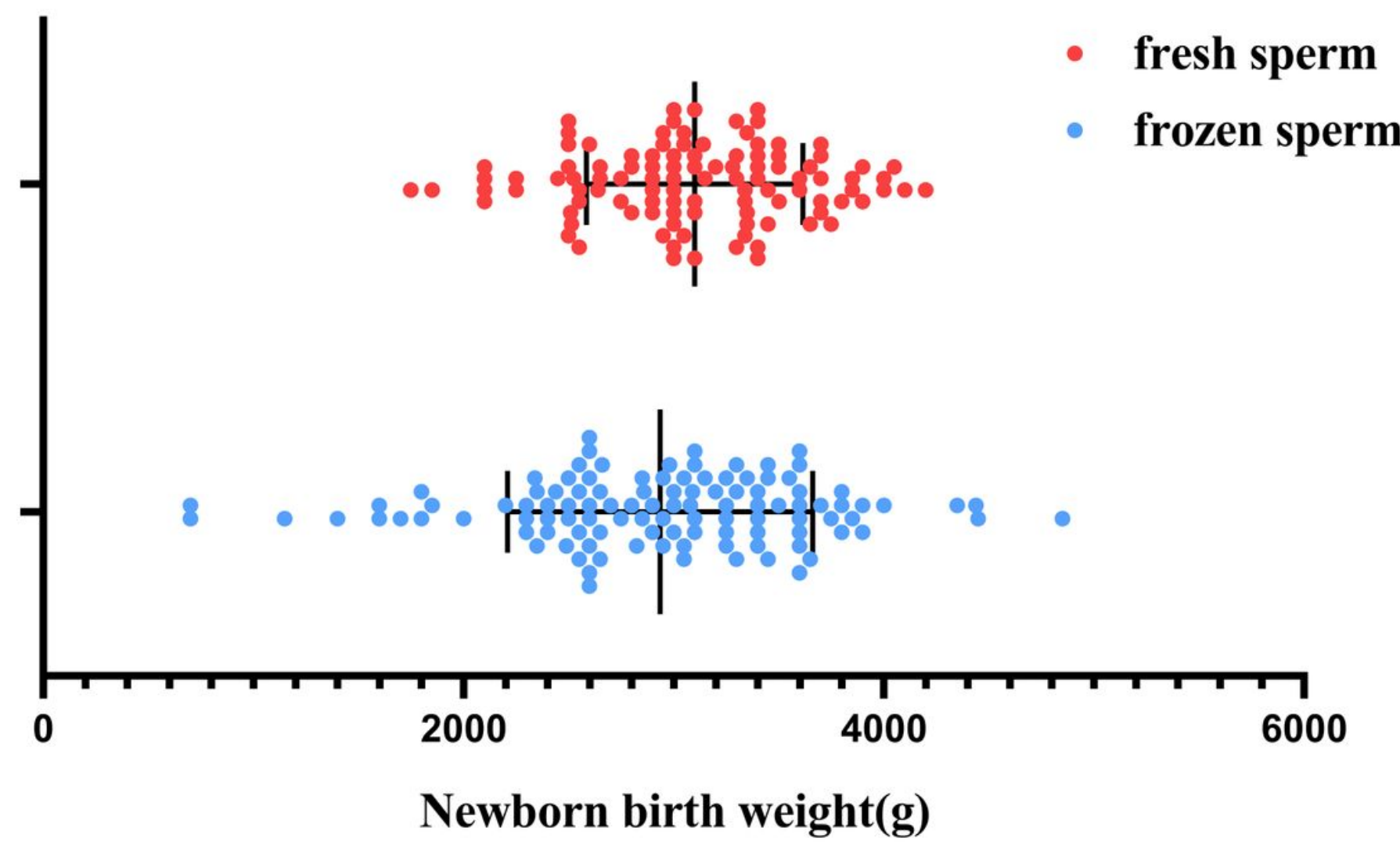

Figure 2

110 babies were born in the frozen sperm group, including 23 low birth weight newborns and 5 macrosomias. 106 babies were born in the fresh sperm group, including 9 low birth weight newborns, and 4 cmacrosomias. The average newborn birth weight(g) in frozen(2932.61 \pm 728.40$)$ versus fresh sperm group(3100.32 \pm 515.64$), P>0.05$. The rate of Low-Birth-Weight newborn in frozen(20.91\%) versus fresh sperm group(8.49\%), $\mathrm{P}<0.05$. 